\title{
Lighting up ATP in cells and tissues using a simple aptamer-based fluorescent probe
}

\author{
Wenjun Liu ${ }^{1} \cdot$ Xuena Zhu ${ }^{2,3} \cdot$ Maedeh Mozneb ${ }^{3} \cdot$ Larry Nagahara $^{4} \cdot$ Tony Y. Hu ${ }^{5} \cdot$ Chen-Zhong Li, ${ }^{3,5}$
}

Received: 15 June 2021 / Accepted: 28 August 2021 / Published online: 23 September 2021

(c) The Author(s), under exclusive licence to Springer-Verlag GmbH Austria, part of Springer Nature 2021

\begin{abstract}
Extracellular ATP as a purinergic signaling molecule, together with ATP receptor, are playing an important role in tumor growth, therapy resistance, and host immunity suppression. Meanwhile ATP is a crucial indicator for cellular energy status and viability, thus a vital variable for tissue regeneration and in vitro tissue engineering. Most recent studies on COVID-19 virus suggest infection caused ATP deficit and release as a major characterization at the early stage of the disease and major causes for disease complications. Thus, imaging ATP molecule in both cellular and extracellular contexts has many applications in biology, engineering, and clinics. A sensitive and selective fluorescence "signal-on" probe for ATP detection was constructed, based on the base recognition between a black hole quencher (BHQ)-labeled aptamer oligonucleotide and a fluorophore (Cy5)-labeled reporter flare. The probe was able to detect ATP in solution with single digit $\mu \mathrm{M}$ detection limit. With the assistance of lipofectamine, this probe efficiently entered and shined in the model cells U2OS within $3 \mathrm{~h}$. Further application of the probe in specific scenery, cardio-tissue engineering, was also tested where the ATP aptamer complex was able to sense cellular ATP status in a semi-quantitative manner, representing a novel approach for selection of functional cardiomyocytes for tissue engineering. At last a slight change in probe configuration in which a flexible intermolecular A14 linker was introduced granted regeneration capability. These data support the application of this probe in multiple circumstances where ATP measurement or imaging is on demand.
\end{abstract}

Keywords Fluorescent probe $\cdot$ Aptamer $\cdot$ ATP $\cdot$ Live cell imaging $\cdot$ Cardiac tissue engineering $\cdot$ COVID-19

\section{Introduction}

Among many metabolites, ATP is majorly produced in mitochondria and resides in cells with a concentration of 5-10 mM. Intracellular ATP represents the principal source

Wenjun Liu and Xuena Zhu contributed equally to this work.

Xuena Zhu

xzhu09@zju.edu.cn

Tony Y. Hu

tonyhu@tulane.edu

$\triangle$ Chen-Zhong Li

chenzhongbiosensor@gmail.com

1 Zhejiang Provincial Key Laboratory of Pancreatic Disease and Department of Hepatobiliary and Pancreatic Surgery, the First Affiliated Hospital, Zhejiang University School of Medicine, Hangzhou 310002, Zhejiang, China

2 Department of Pathology, the First Affiliated Hospital and School of Medicine, Zhejiang University, Hangzhou 310002, Zhejiang, China of cellular energy and is indicative for cell energy status and viability. It is shown to be crucial for in vivo tissue repair/ regeneration and in vitro tissue engineering [1]. On the other hand, extracellular ATP functions as a pivotal signaling mediator [2-4]. Under normal physiological conditions, ATP is barely detectable in extracellular contents. ATP is

3 Nanobioengineering/Bioelectronics Laboratory, Department of Biomedical Engineering, Florida International University, 10555 West Flagler Street, Miami, FL 33174, USA

4 Department of Chemical and Biomolecular Engineering, Whiting School of Engineering, Johns Hopkins University, Baltimore, MD 21218, USA

5 Center for Cellular and Molecular Diagnostics, Department of Biochemistry and Molecular Biology, Tulane University School of Medicine, New Orleans, LA 70112, USA 
profoundly released from cells upon mechanical injury and other stimulus $[5,6]$. ATP enrichment is widely found in tumor microenvironment at up to tens to hundreds of micromolar [7]. ATP and adenosine arised from the hydrolysis of ATP by membrane bound phosphohydrolase CD73 and CD39 are the major purinergic signaling molecules that pose significant influence on the tumor-host interaction, thus contributing to enhanced immunosuppression, tumor growth, and invasion [8-10].

Most recent research revealed that SARS-CoV2 virus (COVID-19) infection causes cellular ATP deficit [11, 12] and ATP release from infected cells, which, dependently on the site of infection, in turn signals unproductive response at lung [13] or leads to neuroinflammation caused complications [14]. For these considerations, a reliable, safe, and live imaging compatible ATP probing system can be conceivably beneficial for laboratory research, engineered tissue growth in vitro, and patient care on clinical sites.

In situ bioimaging has become a powerful tool in bioanalysis [15-18]. Significant progress has been achieved in the sensing and imaging of metabolites in biological samples containing live cells and tissue [19-21]. Until now, diverse methods have been developed to detect ATP, such as traditional approaches (HPLC, NMR spectroscopy [22], capillary electrophoresis [23]), optical sensors (colorimetric assays [24], fluorometric sensors [25], bioluminescence, and chemiluminescence detection platforms [26]), and electrochemical detection methods [26]. However, new testing that is straightforward, bio-friendly, and live imaging compatible remains on demand.

Over the years, a large repository of nucleic acid-based aptamers that possess biomolecular specificity has been selected and reported. Along with it, extensive efforts have been spent on developing aptamer-based sensors ("aptasensors") for the detection of adenosine, ATP [27], cocaine [28], $\mathrm{Hg}^{2+}$ [29], toxins [30], thrombin, vascular endothelial growth factor (VEGF) [31], etc. In the sensing process, aptamers often bind to their targets by a mechanism in which they undergo considerable conformational changes that can be used for converting the binding event into a measurable signal. Combined with various transducers, multiple sensing strategies have been developed, such as fluorescence, colorimetry, electrophoresis, electrochemistry, electrochemiluminescence (ECL), quartz crystal microbalance (QCM), and surface plasmon resonance (SPR) [32-34]. Through direct modification with fluorophores, the conformational changes of aptamers can affect the fluorescence of a dye or the fluorescence resonance energy transfer (FRET) between two dyes. The signal change, either increase (i.e., the "signal-on" mode) or decrease (i.e., the "signal-off" mode), reflects the extent of the binding process, thereby allowing for quantitative measurement of target concentration. Fluorescent molecular probes have so far been playing a major role for intracellular sensing and imaging $[35,36]$. Their fast response, intense signal, relatively low intrinsic cell autofluorescence, and relatively simple instrumental set-up have made fluorescence coupled molecular probe a perfect match for real time measurements in cells.

In this manuscript, we report an easy to fabricate "signalon" fluorescent probe for ATP detection, which is based on hybridization between a black hole quencher (BHQ)-labeled aptamer oligonucleotide and a fluorophore (Cy5)-labeled reporter flare. This simple design functions stand-alone and is free of bulky attachment such as protein enzyme or nanoparticles. Oligonucleotides undergo clearance by both endonucleases and exonucleases in nucleus and cytosol after extended cellular retainment and thus render minimal interference to cell physiology. Cell friendly excitation and emission of red fluorophore represent another advantage of this probe for cellular application. We demonstrate that the probe is of high selectivity and low detection limit for analytical use in solution. In addition, the feasibility of the probe to be used in cellular context is demonstrated by probing intracellular ATP in U2OS cells as well as in neonatal rat cardiomyocytes.

\section{Materials and methods}

\section{Chemicals and materials}

Adenosine 5'-triphosphate (ATP), guanosine 5'-triphosphate (GTP), uridine 5'-triphosphate (UTP), cytidine 5'-triphosphate (CTP), and oligomycin were purchased from SigmaAldrich (St Louis, MO, USA). Imaging compatible Nunc ${ }^{\mathrm{TM}}$ Lab-Tek $^{\text {TM }}$ II 8-well chamber slides (Cat\#: 155409PK), Hoechst 33,342 (Cat\#: H3570), Tubulin Tracker ${ }^{\mathrm{TM}}$ Green (Oregon Green ${ }^{\mathrm{TM}} 488$ Taxol, Cat\#: T34075), neonatal rat cardiomyocyte isolation enzymes, supplements, and primary cell isolation medias were purchased form Thermo Fisher Scientific (Waltham, MA). Neonatal rat hearts were generously donated from FIU's animal facility. All the other chemicals were of analytical reagent grade and were used as received without further purification.

\section{Preparation of aptamer-based ATP probe}

The BHQ-labeled ATP aptamer (5'-ACCTGGGGGAGT ATTGCGGAGGAAGGTGTCACA-BHQ-3') Cy5-labeled reporter oligo (5'-Cy5-TGTGACACCTTCCT-3') (Fig. 1) and the modified probe with A14 linker (Fig. 6A) were synthesized by Integrated DNA Technologies (Coralville, IOWA, USA). A $20 \mu \mathrm{L}$ black hole quencher (BHQ) $(100 \mu \mathrm{M})$ labeled aptamer oligonucleotides were mixed with $20 \mu \mathrm{L}$ fluorophore (Cy5)-labeled reporter strands (100 $\mu \mathrm{M})$ (1:1) in $40 \mu \mathrm{L} 1 \mathrm{X}$ PBS (total: $80 \mu \mathrm{L}$ ), heated to $80^{\circ} \mathrm{C}$, slowly 
Fig. 1 Design and detection mechanism of aptamerbased ATP probes. The probe comprises of two elements, a 3' black hole quencher (BHQ) attached ATP aptamer (ssDNA) and a 5' Cy5 labeled reporter strand. The strands hybridize and present the fluorophore at quenched state when ATP is absent. When ATP incorporates into the molecule complex, conformational change occurs at the aptamer that allows invasion of $5^{\prime}$ end, partial hybridization with $3^{\prime}$ end, and displacement of the reporter flares

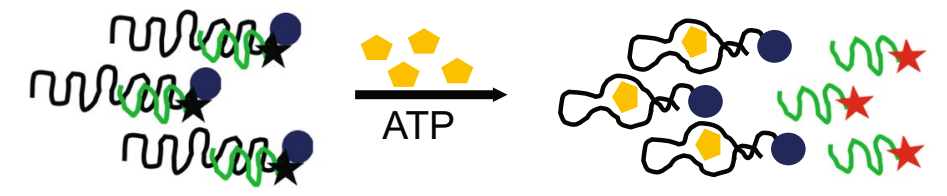

3' BHQ Aptamer-5' Cy5 Reporter Duplexes

Released Reporter Flares

Aptamer: 5' - ACCTGGGGGAGTATTGCGGAGGAAGGTGTCACA - BHQ-3' Reporter: 3'- TCC TTCCACAGTGT - Cy5-5'

cooled to room temperature, and stored in the dark for overnight allowing efficient hybridization. These stock ATP probes $(25 \mu \mathrm{M})$ were stored in the refrigerate $\left({ }^{\circ} \mathrm{C}\right)$ and dark before use.

\section{Fluorescence studies in solution}

Aptamer probes were diluted to a concentration of $250 \mathrm{nM}$ or indicated in 1X PBS. All of the fluorescence spectra were recorded in room temperature immediately after mixing 100 $\mu \mathrm{L}$ diluted probes and ATP samples on a Jasco FP-6500 fluorescence spectrometer with excitation at $633 \mathrm{~nm}$ and emission from 650 to $750 \mathrm{~nm}$ in $1 \mathrm{~nm}$ increments. Optimum temperature for this assessment has not been evaluated. Fluorescence response emerges immediately post-manual mixing, which takes about $2 \mathrm{~s}$, and keeps relatively steady within a 2-h time window. Concentration of the aptamer probe in final reaction can be adjusted to accommodate analytes of different concentration ranges, e.g., $250 \mathrm{nM}$ probe for $1-100$ uM ATP and $2 \mu \mathrm{M}$ probe for $0.1-3 \mathrm{mM}$ ATP. The regeneration study was carried out particularly in nuclease free water and $1 \mathrm{X}$ Apyrase buffer that contain calcium.

\section{Gel electrophoresis and detection by fluorescence}

Fifteen percent native polyacrylamide gels were employed to separate aptamer duplex and free reporter flare based on their difference in mobility. The casting includes $1 \mathrm{X}$ TAE, 15\% acrylamide/Bis 29:1, $50 \mu \mathrm{L} \mathrm{10 \%} \mathrm{ammonium} \mathrm{persul-}$ fate (APS), and $5 \mu \mathrm{L}$ tetramethylethylenediamine (TEMED) for each gel $(6 \mathrm{~mL})$. Electrophoresis was done within miniprotean electrophoresis system. Images were recorded with BioRad ChemiDoc Imaging System by using its Cy5 fluorescence channel. For visualization of release of the Cy5labeled reporter flare in response to ATP by polyacrylamide gel electrophoresis, black hole BHQ quencher was excluded so that the Cy5 fluorescence can be used to trace both free reporter flare and assembled aptamer duplex. A $15 \mu \mathrm{L}$ reaction (Fig. 2D, lanes 3-7) and $3.75 \mathrm{nmol}$ (Fig. 2D, lane 1-2) were loaded for each lane which suffices the detection limit of the ChemiDoc system. The PAGE was run at the voltage of $20 \mathrm{~V}$ for $90 \mathrm{~min}$.

\section{U20S cell culture and imaging}

U2OS cells were incubated in DMEM supplemented with $10 \%(\mathrm{v} / \mathrm{v})$ fetal bovine serum and $1 \%(\mathrm{w} / \mathrm{v})$ penicillin/streptomycin at $37{ }^{\circ} \mathrm{C}$ in the presence of $5 \% \mathrm{CO}_{2}$ in a benchtop incubator. Cells were passaged when they are approximately $80 \%$ confluence. Then, the cells were seeded in a Nunc ${ }^{\mathrm{TM}}$ Lab-Tek ${ }^{\mathrm{TM}}$ II 8 -well chamber slide at a density of $10^{4}$ per well and total media volume $300 \mu \mathrm{L}$. After $24 \mathrm{~h}$, the cells were washed with PBS and replenished with fresh media. A $75 \mu \mathrm{L}$ probes/lipofectamine complex was added to each well. Two hours after adding probes, the cells were washed once with PBS, replenished with fresh media, and subjected to fluorescence microscopic studies. Counterstaining was done by adding tubulin tracker green and Hoechst at final concentration of $0.3 \mu \mathrm{g} / \mathrm{mL}$ and $5 \mu \mathrm{g} / \mathrm{mL}$ respectively, $30 \mathrm{~min}$ before washing. Oligomycin at the final concentration of $10 \mu \mathrm{g} / \mathrm{mL}$ was added to cells $2 \mathrm{~h}$ in prior to probes. Images from green, red, and blue channels were separately shot with $40 \times$ magnitude. Quantification and stacks were prepared with ImageJ.

\section{Cardiomyocyte isolation, culture, and imaging}

Neonatal rat cardiomyocytes were isolated following Thermos Fisher Scientific isolation protocol from newborn (1 2 day old) rat pups. Briefly, the neonatal rats were euthanized via decapitation, and their hearts' ventricles were excised. Each ventricle was immediately put in a 
Fig. 2 The ATP probe responses to ATP in a specific and sensitive manner. A Solution fluorescence spectra of the aptamer-based probe treated with $100 \mu \mathrm{M}$ ATP, UTP, GTP, CTP, and AMP. B Fluorescence spectra of the aptamer-based ATP probe in solution with increasing concentrations of ATP $(0-480 \mu \mathrm{M})$. Note: plotted in $(\mathbf{A})$ and $(\mathbf{B})$ are averages of experimental triplicates. C The peak intensity as a function of ATP concentration. D Visualization of release of the Cy5-labeled reporter flare in response to ATP by polyacrylamide gel electrophoresis and fluorescence imaging. Black hole quencher was excluded to allow detection of the duplex configuration. A $250 \mathrm{nM}$ preformed aptamer-reporter flare duplex was exposed to serially diluted ATP and GTP $(0,16,50$, 150 , and $450 \mu \mathrm{M}$; lanes 3-7), which gave rise to low-position (high mobility) bands that correspond to the free Cy5-labeled reporter flare
A

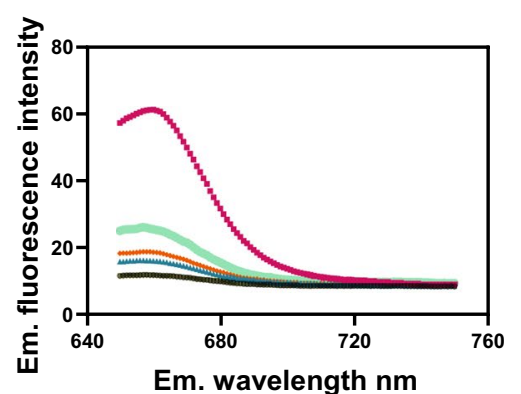

B

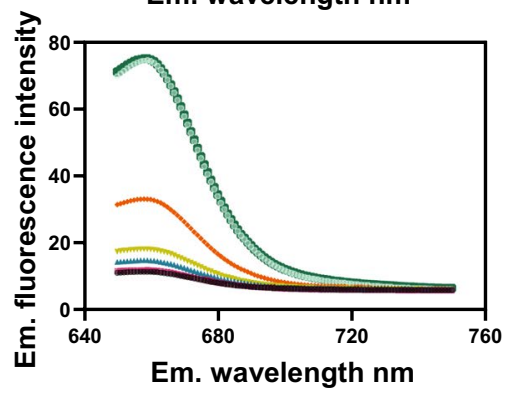

\section{C}

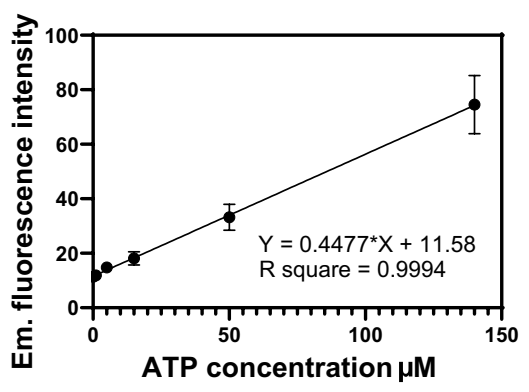

- $250 \mathrm{nM}$ probe

- $250 \mathrm{nM}$ probe +1 uM ATP

- $250 \mathrm{nM}$ probe +5 uM ATP

$250 \mathrm{nM}$ probe +15 uM ATP

- $250 \mathrm{nM}$ probe + 50 uM ATP

$250 \mathrm{nM}$ probe +140 uM ATP

- 250 nM probe +480 uM ATP

- $250 \mathrm{nM}$ probe

- probe $+100 \mu \mathrm{M}$ ATP

$\Delta$ probe $+100 \mu \mathrm{M}$ GTP

probe $+100 \mu \mathrm{M}$ CTP

probe $+100 \mu \mathrm{M}$ UTP

probe $+100 \mu \mathrm{M}$ AMP
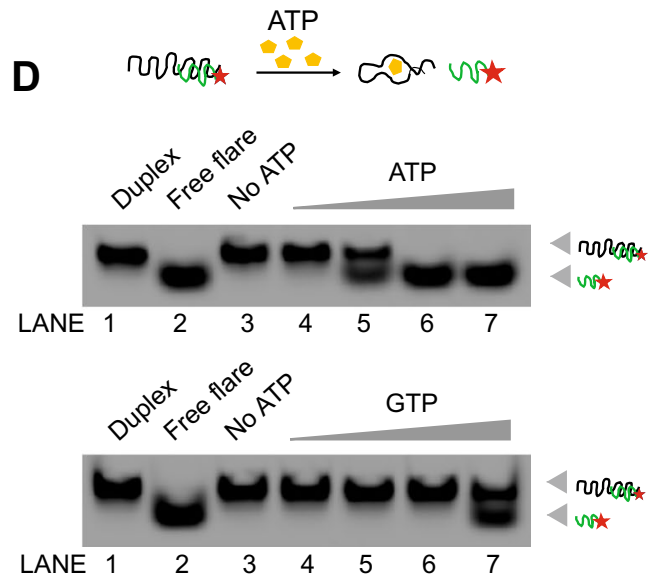

separate $1.5-\mathrm{mL}$ vial filled with ice cold HBSS. They were then trimmed into small pieces $\left(1-3 \mathrm{~mm}^{2}\right)$ with surgical scissors. A $0.2 \mathrm{~mL}$ reconstituted cardiomyocyte isolation enzyme 1 (papain) in HBSS and $10 \mu \mathrm{L}$ cardiomyocyte isolation enzyme 2 (thermolysin) were added into each tube to digest the cardiac tissue. Vials were incubated in a benchtop incubator $\left(37{ }^{\circ} \mathrm{C}\right.$ and $\left.5 \% \mathrm{CO}_{2}\right)$ for $35 \mathrm{~min}$. The digests were then collected, and complete DMEM (with $1 \%$ penicillin/ streptomycin, $10 \%$ fetal bovine serum (FBS)) was added to each vial to stop enzyme activity. By using a 1000-unit pipette, the tissue was broken up (pipetting up and down for 30 times until tissue was a single-cell suspension). The vials were combined for cell concentration and viability determination. Finally, the cells were seeded in an 8-well chamber slide at a density of $10^{4}$ cells per well with complete DMEM for primary cell isolation (total media volume 300 $\mu \mathrm{L}$ ). After $24 \mathrm{~h}$, the complete DMEM was substituted with media containing cardiomyocytes growth supplement, for cardiomyocyte purification over fibroblasts and endothelial cells. After this, the medium was changed every 3 days. At day 7 , the isolated cardiomyocyte was matured enough for following experiments. Staining of aptamer probes, tubulin tracker green, and Hoechst were done in the same way as for U2OS cells.

\section{ATP sensor enters cells with the assistant of lipofectamine}

Cellular delivery of ATP sensor is mediated by lipofectamine. The positive surface charge of the liposomes mediates the interaction of the oligonucleotide and the cell membrane, allowing for fusion of the liposome/oligonucleotide transfection complex with the negatively charged cell membrane and thus successful entry of ATP sensors. In brief, equal volume of diluted probe $(0.33 \mu \mathrm{M})$ in serumfree media and $1.25 \%$ lipofectamine diluted in serum-free median was mixed to form the probe/lipofectamine complex. This sensing complex was added to cell culture (in 1:4 ratio) 30 min after mixing. 


\section{Flow cytometry}

For flow cytometry studies, cells were seeded in 24 well plates at $50 \mathrm{k} / \mathrm{well}$. The next day, $100 \mu \mathrm{L}$ probe/lipofectamine complex or vehicle control was added into 400 $\mu \mathrm{L}$ culture media. After $2 \mathrm{~h}$ incubation in $37^{\circ} \mathrm{C}$, cells were washed with $1 \mathrm{X}$ PBS, trypsinized and resuspended in 500 $\mu \mathrm{L}$ complete media, and transferred to flow cytometry sample tubes. Samples were subjected to BD FACS Canto-II analysis immediately with configuration ex $640 \mathrm{~nm}$, em $660 / 20 \mathrm{~nm}$ for Cy5 resolution. Time course evaluation was carried out at $0,0.5,1,2,3 \mathrm{~h}$ post addition of probe/lipofectamine complex. It was determined that $2 \mathrm{~h}$ incubation be used for other flow and imaging studies. Specificity of the probes to cellular ATP was verified with preceding $2 \mathrm{~h}$ of $1 \mu \mathrm{g} / \mathrm{mL}$ oligomycin treatment.

\section{Results and discussion}

\section{ATP-specific aptamer-based fluorescence probe exhibits high sensitivity and broad detection range in solution}

As illustrated in Fig. 1, our aptamer-based ATP probe duplex is composed of a $3^{\prime}$ BHQ-labeled single strand DNA aptamer and a 5' Cy5-labeled reporter strand representing an extremely simple design. With the presence of ATP molecules, the aptamer strand undergoes conformation change that facilitates self-annealing of its $5^{\prime}$ and $3^{\prime}$ ends and along with it the displacement of the reporter flare. The release of reporter flare is detected by a fluorometer for quantification of ATP abundance.

Aptamer-based probe duplexes showed a significant fluorescence increase upon target addition. For $250 \mathrm{nM}$ probe, when $100 \mu \mathrm{M}$ ATP was added, an immediate 6.56fold increase in fluorescent signal was observed (Fig. 2A). Conversely, addition of the ATP analogues guanidine triphosphate (GTP), cytosine triphosphate (CTP), or uridine triphosphate (UTP) at $100 \mu \mathrm{M}$ did not result in a significant increase in fluorescent signal when compared to $100 \mu \mathrm{M}$ ATP (Fig. 2A). Adenosine monophosphate (AMP) results in the largest fluorescence increase among these analogues. These results are consistent with a selective target binding ability of the aptamer probe toward adenosine, and the probe is most responsive to ATP.

The effect of target concentration was also investigated. Probe duplexes $(250 \mathrm{nM})$ responded to the presence of ATP in a dose-dependent manner (Fig. 2B) with a linear range up to $140 \mu \mathrm{M}$ ATP (Fig. 2C) demonstrating that the fluorescence can be used to quantify ATP concentration in solution. Native polyacrylamide gels were utilized to resolve each reaction species (Fig. 2D). With an aptamer chain that does not contain BHQ group, Cy5 fluorescence assisted in identifying of both assembled probe (lane 1) and free reporter flare (lane 2). ATP concentration-dependent dissociation of the reporter flares from assembly was reproduced on native PAGE as well (lanes 3-7).

Meanwhile, the detection range of the probe in solution can be probe concentration dependent. It is capable of sensing high concentration ATP by simply increasing the concentration of the probe. For instance, the probe at the concentration of $2 \mu \mathrm{M}$ was responsible to ATP at hundreds $\mu \mathrm{M}$ to single digit $\mathrm{mM}$ (Fig. S1). The broad detection limit (at least single digit $\mu \mathrm{M}$ to $\mathrm{mM}$ ) supports its usage in both analytical measurements where only trace amount of ATP is present and live cell ATP imaging when physiological ATP concentration elevates to $\sim 5 \mathrm{mM}$.

\section{Study with model cell U20S: the ATP aptamer-based probe senses intracellular ATP and facilitates live cell imaging}

Having demonstrated the signaling ability of the aptamerbased ATP probe in solution, we next tested its ability to detect intracellular ATP. Oligonucleotides do not enter cell actively. One of the widely used strategies to facilitate cellular delivery of oligonucleotides is to use liposome-based carriers as illustrated in Fig. S2. We evaluated a few commercially available liposome acting reagents and determined to use lipofectamine for following studies due to its relatively high efficiency for the probe.

Model cell line U2OS (human bone osteosarcoma) was used to evaluate the entry and performance of the probe. At first, flow cytometry was employed to monitor the emerging of Cy5 positive cell population over a 3-h time course (Fig. 3). An evident shift toward Cy5 positive population was observed at $1 \mathrm{~h}(53.6 \%)$ that reaches $91.8 \%$ at $3 \mathrm{~h}$ (Fig. 3A and $\mathrm{C}$ ). To confirm that the release of $\mathrm{Cy} 5$ fluorophore inside cells is specific to ATP, we treated cells with oligomycin that blocks ATP synthesis and thus causes depletion of cellular ATP. Addition of oligomycin undermined the release of Cy5 leading to a drop of Cy5 positive population from 91.8 to $44 \%$ suggesting that the probe is specific to intracellular ATP concentration (Fig. 3B and C). Two hours was used as standard treatment for following studies. Moreover, efficient cellular uptake by two more cell types HEK-293 and MEF were observed (Fig. S3A and S3B) suggesting wide applicability of this ATP probe for a broad spectrum of cell types.

The capability of sensing intracellular ATP in cellular context is further evaluated. Imaging is the most adoptable approach for sensing of intracellular molecules in live cells. As a result, we evaluated the compatibility and performance of the probe in fluorescent live cell imaging. Perinuclear and cytosolic localization of released $\mathrm{Cy} 5$ was observed $2 \mathrm{~h}$ after the administration of the probe (Fig. 4A 
Fig. 3 Aptamer-based ATP probe senses cellular ATP in U2OS model cell. A Time course flow cytometry facilitated profiles of $\mathrm{Cy} 5$ release post-lipofectamine-assisted delivery of the probe. Considerable right (Cy5 positive) shift started to occur shortly at $1 \mathrm{~h}$ and reached close-to-completion state at $3 \mathrm{~h}$. Two hours is used for all following investigation. B The release of Cy5 and fluorescence turn-on was specific to ATP as oligomycin pretreatment largely abrogated the right shift. C Statistics of U2OS cell fluorescence at each time points of probe delivery
A

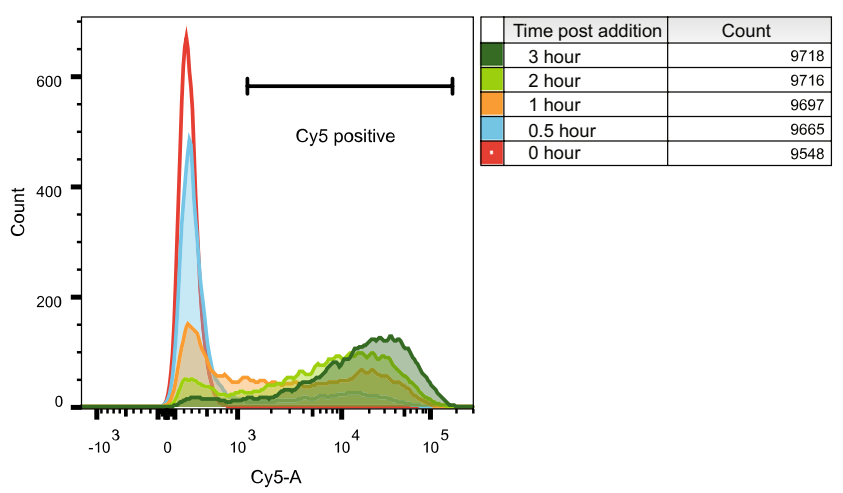

B

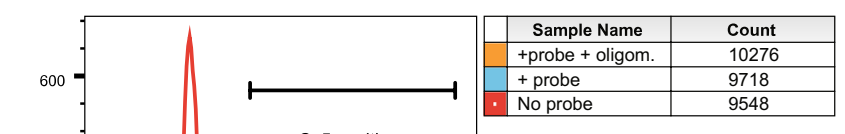

C

\begin{tabular}{|l|l|l|l|l|}
\hline Sensor treatment & \multicolumn{1}{c|}{$\begin{array}{c}\text { Cy5 positive } \\
\text { Freq. of Parent }\end{array}$} & Mean & Median & SD \\
& & & & \\
\hline 3 hour + oligo. & 44.0 & 8990 & 715 & 16774 \\
\hline 3 hour & 91.8 & 28956 & 19499 & 29691 \\
\hline 2 hour & 80.7 & 16738 & 9063 & 20641 \\
\hline 1 hour & 53.6 & 10270 & 2131 & 16820 \\
\hline 0.5 hour & 18.2 & 3278 & 369 & 9173 \\
\hline 0 hour & 0.021 & 293 & 278 & 132 \\
\hline & & & & $\mathbf{1 6 9 0 0}$ \\
\hline Mean & $\mathbf{5 1 . 1}$ & $\mathbf{1 2 3 6 8}$ & $\mathbf{5 6 2 7}$ & $\mathbf{9 8 7 6}$ \\
\hline SD & $\mathbf{3 3 . 2}$ & $\mathbf{9 7 5 4}$ & $\mathbf{7 0 6 8}$ & \\
\hline
\end{tabular}

(top)), while oligomycin treatment reduced the staining intensity (Fig. 4A (below). ImageJ facilitated quantification of fluorescence (Fig. 4B) suggests a statistically significant diminish with ATP depletion by oligomycin. These data are also in agreement with the flow cytometry experiments (Fig. 3B). In summary, the probe enters the model cells U2OS with high efficiency shortly within $3 \mathrm{~h}$. The probe is live cell imaging compatible and possesses high specificity for intracellular ATP. Moreover, the probe is intrinsically low in cytotoxicity (Fig. S4). Uptaken probes eventually undergo nuclease mediated clearance and metabolization. As cyanine fluorophores is known to possess low toxicity [37], we did observe disappearance of cellular fluorescence over $48 \mathrm{~h}$ (data not shown). Unlike nanoparticle mediated delivery which deposits particles to both cells and culture, lipofectamine is considerably cell friendly.

\section{ATP aptamer-based probe assists in characterization and selection of functional cardiomyocyte isolates}

U2OS serves as a representative of cultured cells with relatively high homogeneity. Intracellular molecular sensing for extreme cellular/tissue context represents the highest challenge in the field of sensor development. We were prompted to evaluate the sensing performance of the probe in isolated cardiomyocyte culture, a crucial material for cardiac tissue engineering.

Neonatal rat heart was harvested and subject to isolation and selection for cardiomyocytes. After the procedure functional colonies when assessed by spontaneous beating comprises $\sim 20 \%$ of the all population (data not shown). Flow cytometry suggested $37.5 \%$ Cy 5 positive cells after $2 \mathrm{~h}$ probe staining (Fig. 5A). The overall elevation of fluorescence was statistically significant (Fig. 5A statistic panel). 
Fig. 4 Live cell imaging with the ATP probe. A Fluorescence images of ATP probe-stained live cells without (top) or with (below) the treatment of oligomycin $(10 \mu \mathrm{g} / \mathrm{mL})$. Red, ATP probe; green, tubulin (cytosol); blue, Hoechst (nuclei). Cytosolic and perinuclear fluorescence of ATP probe was evidently observed $2 \mathrm{~h}$ post the introduction of probe-lipofectamine complex. Specificity of ATP probe was validated with oligomycin treatment $(10 \mu \mathrm{g} / \mathrm{mL})$ which suppressed ATP probe signal. B Statistical analysis revealed significant reduction $(p<0.01)$ of ATP probe signal in the oligomycin treated cells. Microscopy fluorescence quantification was carried out with ImageJ. ** denotes significant changes with $p$ value $<0.01$ estimated by $t$-test
A
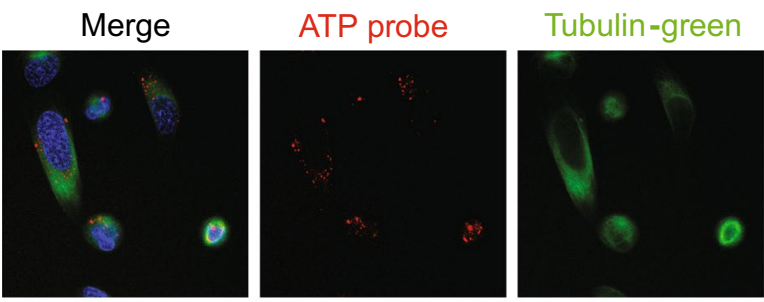

Hoechst

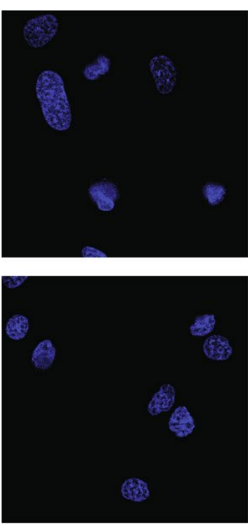

B

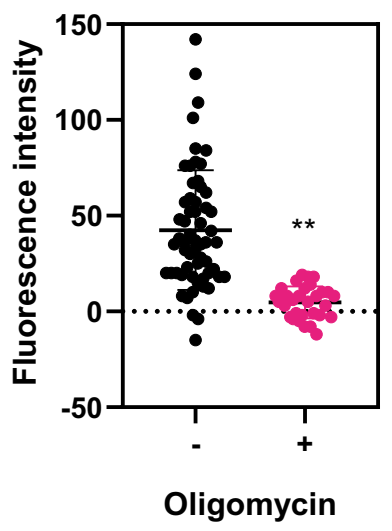

The specificity of the probe was confirmed with reversal (Cy5 positive drops from 37.5 to $15.7 \%$ ) when given oligomycin mediated ATP depletion (Fig. 5A). The "reduced Cy5 positive population" (37.5\%) when compared to U2OS (90.7\%) can be attributed to inherent low transfection efficiency of liposome base approaches for selected cell type and reduced viability post the isolation and selection procedure which is also implied by low percentage of functional colonies. Nevertheless, similar cellular staining pattern was observed as in U2OS cells where perinuclear and cytosolic localization was found (Fig. 5B (top)). Consistent with the flow cytometry study, microscopy staining diminishes alongside with the oligomycin treatment suggesting specificity to ATP (Fig. 5B (below)). Moreover, we also documented match between spontaneous beatings of cardiomyocytes with positive staining (Fig. 5C). While the probe is able to enter cultured cardiomyocyte and perform as expected. We believe that a loss of efficiency in cellular delivery does exist and represents the largest limitation of application of the probe in such cellular context. Studies on more appropriate or distinct delivery procedure are on demand. Nevertheless, the probe represents an efficient and alternative approach to characterize fresh cardiomyocyte isolates that could assist more accurate selection of functional and viable colonies which is crucial for the success of cardiac engineering.

\section{A14 flexible intermolecular link confers regeneration capability}

The reporter flare separates from the ATP aptamer when ATP incorporates into the complex. Diffusion of the released chain makes the probe un-reusable. A rationale approach toward regeneration capability is to introduce a linker between the aptamer and reporter flare molecules. A new probe with additional A14 sequence (Fig. 6A) was synthesized. The Cy5 and quencher groups are repositioned for technical hardship in synthetic chemistry. We validated the ATP sensing ability and regeneration capability of the modified probe in solution with ATP depletion by phosphohydrolase apyrase that converts ATP to AMP which is a poor substrate for the ATP aptamer (Fig. 2A).

The modified probe conserves the ATP sensing ability. Addition of $140 \mu \mathrm{M}$ ATP to $250 \mathrm{nM}$ probe in solution elicits a strong increase in fluorescence (Fig. 6B). However, ATP depletion by apyrase does not render fast turn-over of fluorescence possibly due to a retardant annealing between the 
Fig. 5 Application of the ATP probe in the source materials of cardiac tissue engineering, fresh cardiomyocyte isolate. A Timely cellular uptake assessed by flow cytometry in newly isolated neonatal rat cardiomyocytes. Statistically significant red shift was observed $2 \mathrm{~h}$ after addition of the ATP probe. Oligomycin pretreatment efficiently restrains the red shift. B Microscopy images of ATP probe-stained live cardiomyocytes. Red, ATP probe; green, tubulin (cytosol); blue, Hoechst (nuclei). Cytosolic and perinuclear fluorescence of ATP probe was evidently observed $2 \mathrm{~h}$ post-introduction of the probelipofectamine complex (top). Consistent with the flow cytometry study oligomycin treatment $(10 \mu \mathrm{g} / \mathrm{mL})$ suppresses ATP probe signal in a treatment time dependent manner (below). C Fluorescence images of ATP probe-stained live cardiomyocytes. "Beating" is associated with high ATP concentration as indicated by the ATP probe
A
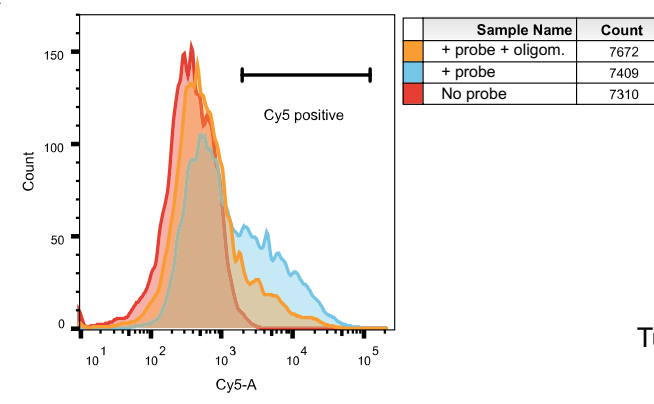

C
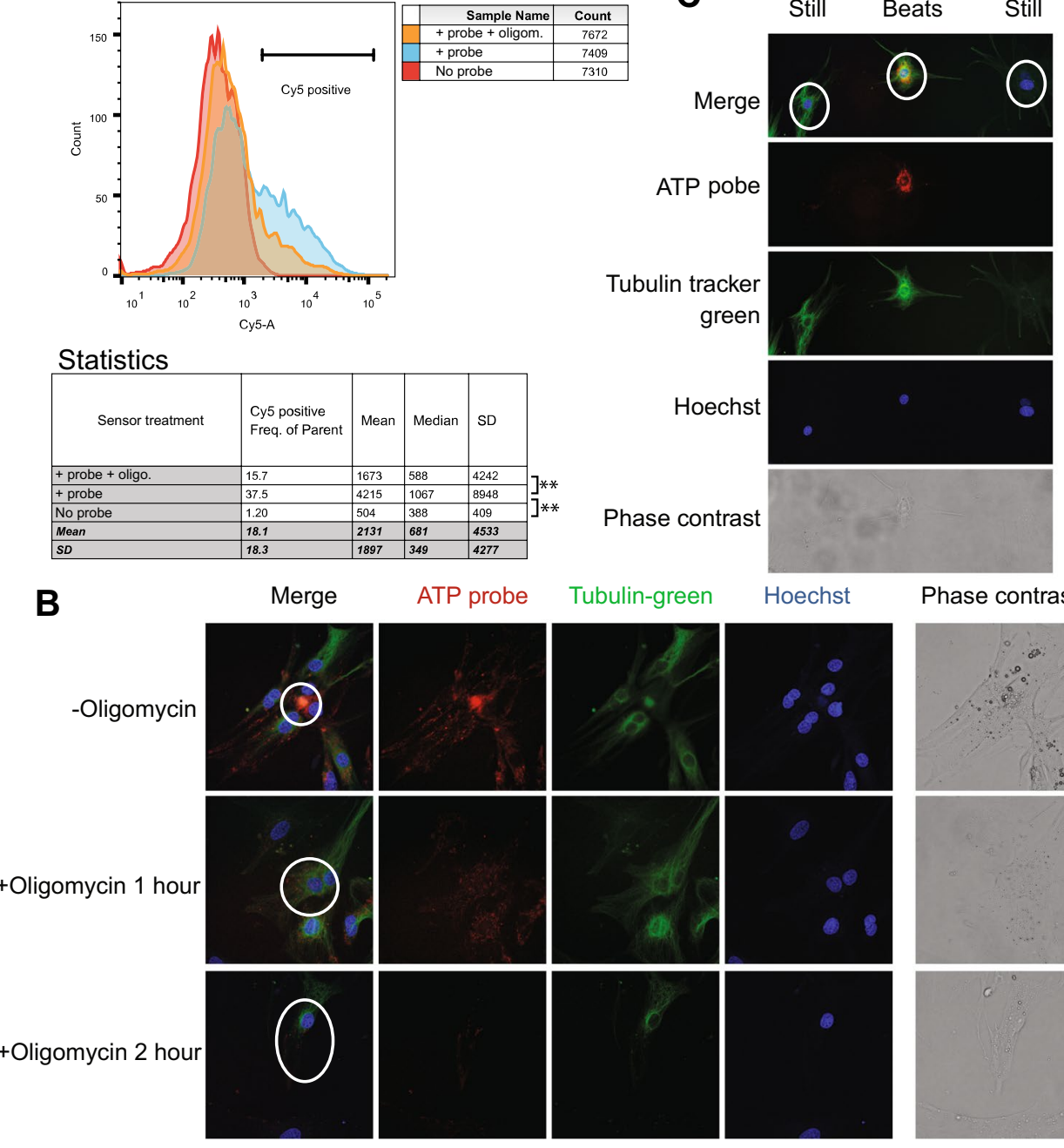

ATP probe

Tubulin-green

Hoechst

Phase contrast
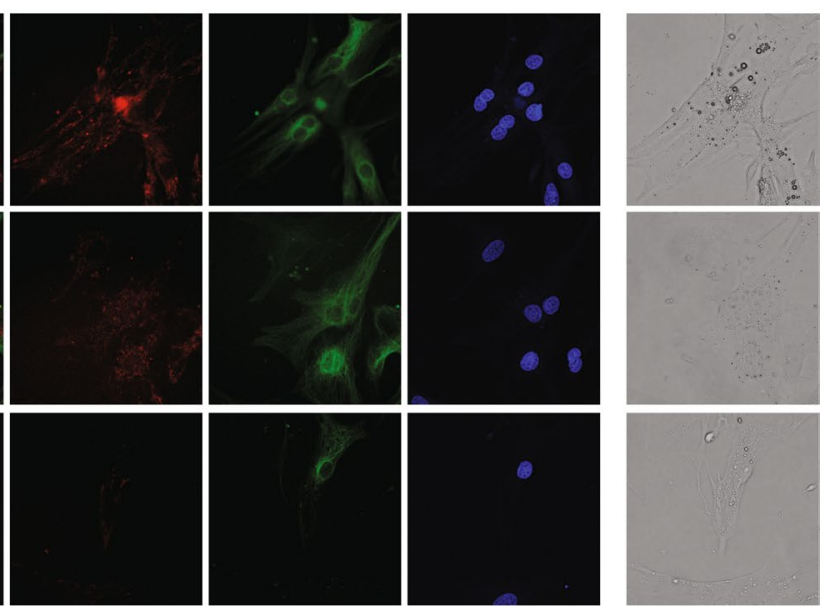

aptamer and reporter chain though the resulting structure theoretically resides thermodynamically more stable. As a result, manned regeneration was given. The calculated melting temperatures ( $\mathrm{Tm}$ ) for the fluorescent and quenched states are calculated as 12 and $42{ }^{\circ} \mathrm{C}$ respectively. The solution was subject to $70{ }^{\circ} \mathrm{C}, 10 \mathrm{~min}$ of incubation, which fully separates the complementary segments, and followed by slow cooling down to room temperature. This allows reanneal and formation of the fluorescence quenched structure as well as inactivation of apyrase activity. Despite a slightly elevated basal fluorescence signal, possibly due to the limited affinity of the probe to AMP, a second addition of ATP induces a pronounced increase in fluorescence (Fig. 6B). These suggest that the new probe configuration with the poly(A) sequence and repositioned fluorophores retains the ATP sensing ability and can be regenerated by simple temperature manipulation.

Introduction of the flexible multiple-adenosine linker between the aptamer and reporter strand restricts the spatial diffusion of the reporter strand post ATP incorporation and makes the probe regeneration compatible. Thorough regeneration relies on temperature manipulation according to our in tube test, which limits the use in a realtime and constant monitoring of ATP changes in solution. Nevertheless, immobilization of the probes on to solid phase could support its use as an on-demand probe such as a multiple-use electrode or a reusable $\mathrm{pH}$ strip. It is worth noting that denaturing at $70{ }^{\circ} \mathrm{C}$ was tested and used instead of more common $95-100{ }^{\circ} \mathrm{C}$ followed by cooling down. This poses reduced impacts on any further modification to the probe, for example, solid phase immobilization which might be sensitive to high temperature. Meanwhile, to be a long lasting reusable sensing platform, further exploration is indeed needed to improve the stability of the probe in vitro. We propose capsulation of the probe into a small molecular permeable outlayer as a protection from environmental nuclease. 
A
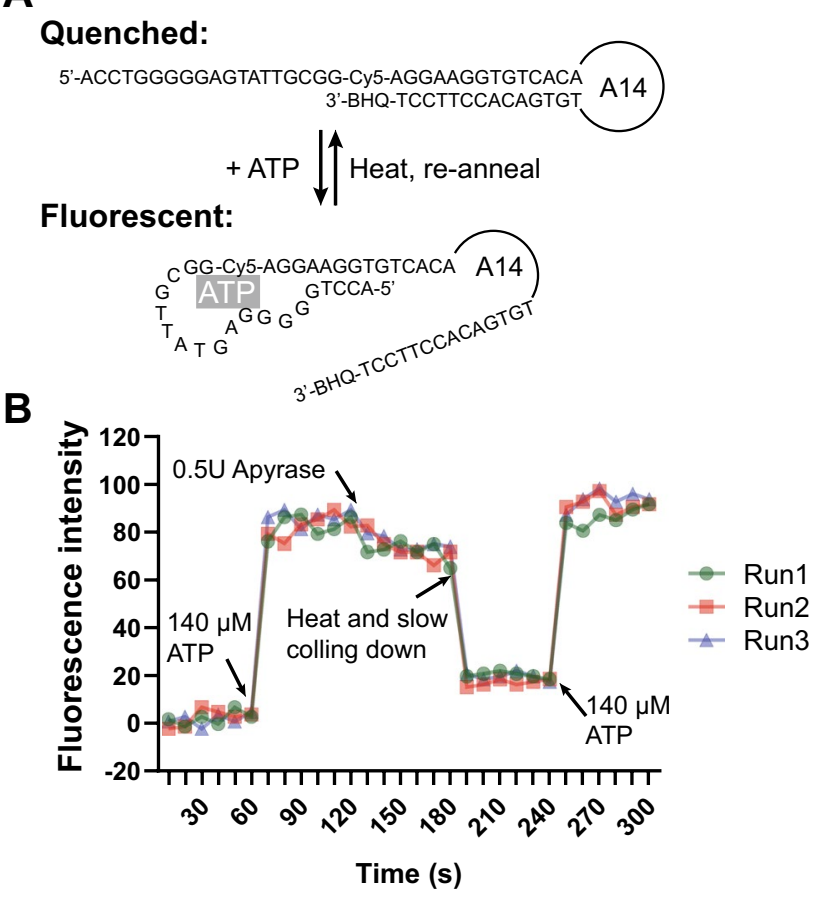

Fig. 6 Reversible ATP aptamer probe and its regeneration. A A 14 adenosine linker is introduced between the ATP responsive aptamer and reporter flare molecules to form the new reversible probe. Switch between the two fluorescent states is realized by increasing ATP or heat and re-anneal. B Functional validation of the new probe is carried out in solution with $250 \mathrm{nM}$ probe and $140 \mu \mathrm{M}$ ATP. All components are reconstituted in nuclease free water. Comparable fluorescence response is retained by the modified probe. Apyrase mediated hydrolysis of ATP results in limited conversion between the states. Nevertheless, sufficient regeneration can be achieved by $70{ }^{\circ} \mathrm{C}$, 10 min heating and slow cooling down, which inactivates the apyrase activity as well. Considerable fluorescence response is restored by this regeneration protocol as a second ATP addition elicits fluorescence considerably. Note that the lapse on time axis is mostly on scale except for the additions of ATP the step of regeneration when the intervals are omitted and presented as $10 \mathrm{~s}$

\section{Conclusion}

We present a new, simple aptamer-based probe that can be used to detect and quantify a small molecule analyte (ATP) in both solution and live cell. The aptamer-based probe is cell friendly and causes limited cytotoxicity as the major components oligonucleotides and cyanine fluorophores can be metabolized by cells. The probe is of high sensitivity to trace level ATP (single digit $\mu \mathrm{M}$, Fig. 2). Given the advantage of not depending on complex conversion of FRET signal, or quench by large molecules/structures (nanoparticle, nanosheets), the probe is of high versatility as differential formations (probe concentration) could be readily adopted for sensing of ATP at broad range concentrations, from $\mu \mathrm{M}$ to physiologically relevant $\mathrm{mM}$ by straightforward changing the probe concentration (Fig. S1) and even higher. With the assistance of lipofectamine, the presented aptamer probe readily enters U2OS cells and is able to directly sense intracellular ATP levels, which makes them well-suited for live cell imaging and sorting studies. In a new rat cardiac isolated which represents a more delicate culture of higher cellular heterogeneity, by sensing intracellular ATP concentration, the probe is able to distinguish functional (beating) colonies. This represents a novel fluorescence-based approach for efficient selection of cardiomyocytes that are the most crucial materials for cardiac tissue engineering.

Supplementary Information The online version contains supplementary material available at https://doi.org/10.1007/s00604-021-05012-8.

Author contribution Xuena Zhu, Wenjun Liu, and Chen-Zhong Li designed the study. Xuena Zhu, Wenjun Liu, and Maedeh Mozneb conducted experiments and collected data. Larry Nagahara analyzed data. Xuena Zhu, Wenjun Liu, and Chen-Zhong Li wrote the article. Tony Y. Hu and Chen-Zhong Li directed and supervised the research.

Funding This work was partially supported by the Engineering Research Centers Program of the National Science Foundation (NSF) under NSF Cooperative Agreement. C.-Z.L. thanks the NSF Independent Research/Development program.

Data availability All requests with regard to data and materials can be addressed to Xuena Zhu, xzhu09@zju.edu.cn; Tony Y. Hu, tonyhu@ tulane.edu; and Chen-Zhong Li, chenzhongbiosensor@gmail.com.

Code availability Not applicable.

\section{Declarations}

Ethics approval All applicable international, national, and/or institutional guidelines for the care and use of animals were followed.

Consent to participate Not applicable.

Consent for publication Not applicable.

Conflict of interest The authors declare no competing interests.

\section{References}

1. Shyh-Chang N, Zhu H, Yvanka de Soysa T, Shinoda G, Seligson MT et al (2013) Lin28 enhances tissue repair by reprogramming cellular metabolism. Cell 155(4):778-92. https://doi.org/10. 1016/j.cell.2013.09.059

2. Patel A, Malinovska L, Saha S, Wang J, Alberti S et al (2017) ATP as a biological hydrotrope. Science 356(6339):753-756. https:// doi.org/10.1126/science.aaf6846

3. McKenney RJ, Huynh W, Tanenbaum ME, Bhabha G, Vale RD (2014) Activation of cytoplasmic dynein motility by dynactincargo adapter complexes. Science 345(6194):337-341. https://doi. org/10.1126/science. 1254198

4. Wu D, Lee S, Luo J, Xia H, Gushchina S et al (2018) Intraneural injection of ATP stimulates regeneration of primary sensory axons in the spinal cord. J Neurosci 38(6):1351-1365. https://doi.org/ 10.1523/jneurosci.1660-17.2017 
5. Mikolajewicz N, Zimmermann EA, Willie BM (2018) Mechanically stimulated ATP release from murine bone cells is regulated by a balance of injury and repair. 7: https://doi.org/10.7554/eLife. 37812

6. Sun D, Junger WG, Yuan C, Zhang W, Bao Y et al (2013) Shockwaves induce osteogenic differentiation of human mesenchymal stem cells through ATP release and activation of P2X7 receptors. Stem Cells 31(6):1170-1180. https://doi.org/10.1002/stem.1356

7. Di Virgilio F, Adinolfi E (2017) Extracellular purines, purinergic receptors and tumor growth. Oncogene 36(3):293-303. https:// doi.org/10.1038/onc.2016.206

8. Kasama H, Sakamoto Y, Kasamatsu A, Okamoto A, Koyama T et al (2015) Adenosine A2b receptor promotes progression of human oral cancer. BMC Cancer 15:563. https://doi.org/10.1186/ s12885-015-1577-2

9. Amoroso F, Capece M, Rotondo A, Cangelosi D, Ferracin M (2015) The P2X7 receptor is a key modulator of the PI3K/GSK3 $\beta /$ VEGF signaling network: evidence in experimental neuroblastoma. Oncogene 34(41):5240-51. https://doi.org/10.1038/onc. 2014.444

10. Yang H, Geng YH, Wang P (2020) Extracellular ATP promotes breast cancer invasion and chemoresistance via SOX9 signaling. 39(35): 5795-5810. https://doi.org/10.1038/s41388-020-01402-z

11. Özilgen M, Yilmaz B (2020) COVID-19 disease causes an energy supply deficit in a patient. Int J Energy Res. https://doi.org/10. 1002/er.5883

12. Taghizadeh-Hesary F, Akbari H (2020) The powerful immune system against powerful COVID-19: A hypothesis. Med Hypotheses 140:109762. https://doi.org/10.1016/j.mehy.2020.109762

13. Edwards C (2020) New horizons: does mineralocorticoid receptor activation by cortisol cause ATP release and COVID-19 complications? J Clin Endocrinol Metab. https://doi.org/10.1210/clinem/dgaa874

14. Ribeiro DE, Oliveira-Giacomelli Á (2020) Hyperactivation of $\mathrm{P} 2 \mathrm{X} 7$ receptors as a culprit of COVID-19 neuropathology. 1-16. https://doi.org/10.1038/s41380-020-00965-3

15. Yu K, Hai X, Yue S, Song W, Bi S (2021) Glutathione-activated DNA-Au nanomachine as targeted drug delivery platform for imaging-guided combinational cancer therapy. Chem Eng J 22:129535. https://doi.org/10.1016/j.cej.2021.129535

16. Yue S, Li Y, Qiao Z, Song W, Bi S (2021) Rolling circle replication for biosensing, bioimaging, and biomedicine. Trends Biotechnol. https://doi.org/10.1016/j.tibtech.2021.02.007

17. Yue S, Song X, Song W, Bi S (2019) An enzyme-free molecular catalytic device: dynamically self-assembled DNA dendrimers for in situ imaging of microRNAs in live cells. Chem Sci 10(6):16511658. https://doi.org/10.1039/c8sc04756a

18. Abe H, Ino K, Li CZ, Kanno Y, Inoue KY et al (2015) Electrochemical imaging of dopamine release from three-dimensionalcultured PC12 cells using large-scale integration-based amperometric sensors. Anal Chem 87(12):6364-6370. https://doi.org/10. 1021/acs.analchem.5b01307

19. Zhang L, Su F, Buizer S, Kong X, Lee F et al (2014) A polymerbased ratiometric intracellular glucose sensor. Chem Commun (Camb) 50(52):6920-6922. https://doi.org/10.1039/c4cc01110d

20. Wu S, Wu S, Yi Z, Zeng F, Wu W et al (2018) Hydrogel-based fluorescent dual $\mathrm{pH}$ and oxygen sensors loaded in 96-well plates for high-throughput cell metabolism studies. Sensors (Basel):18(2). https://doi.org/10.3390/s18020564

21. Li C, Numata M, Takeuchi M, Shinkai S (2005) A sensitive colorimetric and fluorescent probe based on a polythiophene derivative for the detection of ATP. Angew Chem Int Ed Engl 44(39):63716374. https://doi.org/10.1002/anie.200501823

22. Middleton DA, Hughes E, Esmann M (2011) The conformation of ATP within the Na, K-ATPase nucleotide site: a statistically constrained analysis of REDOR solid-state NMR data. Angew Chem Int Ed Engl 50(31):7041-7044. https://doi.org/10.1002/ anie. 201100736
23. Fu C, Fang Y, Song L (1999) Determination of adenosine phosphate by co-electroosmotic capillary electrophoresis with amperometric detection. Anal Chim Acta 399(3):259-263

24. Wang J, Wang LH, Liu XF, Liang ZQ, Song SP et al (2007) A Gold Nanoparticle-Based Aptamer Target Binding Readout for ATP Assay. Adv Mater 19(22):3943-3946

25. Lee DH, Kim SY, Hong JI (2004) A fluorescent pyrophosphate sensor with high selectivity over ATP in water. Angew Chem Int Ed Engl 43(36):4777-4780. https://doi.org/10.1002/anie.20045 3914

26. Perez-Ruiz T, Martinez-Lozano C, Tomas V, Martin J (2003) Determination of ATP via the photochemical generation of hydrogen peroxide using flow injection luminol chemiluminescence detection. Anal Bioanal Chem 377(1):189-194. https://doi.org/ 10.1007/s00216-003-2071-2

27. Nakano S, Mashima T, Matsugami A, Inoue M, Katahira M et al (2011) Structural aspects for the recognition of ATP by ribonucleopeptide receptors. J Am Chem Soc 133(12):4567-4579. https://doi.org/10.1021/ja110725d

28. Du Y, Chen C, Zhou M, Dong S, Wang E (2011) Microfluidic electrochemical aptameric assay integrated on-chip: a potentially convenient sensing platform for the amplified and multiplex analysis of small molecules. Anal Chem 83(5):1523-1529. https://doi. org/10.1021/ac101988n

29. Xu JP, Song ZG, Fang Y, Mei J, Jia L et al (2010) Label-free fluorescence detection of mercury(II) and glutathione based on $\mathrm{Hg} 2+-D N A$ complexes stimulating aggregation-induced emission of a tetraphenylethene derivative. Analyst 135(11):3002-3007. https://doi.org/10.1039/c0an00554a

30. Wang L, Chen W, Ma W, Liu L, Ma W et al (2011) Fluorescent strip sensor for rapid determination of toxins. Chem Commun (Camb) 47(5):1574-1576. https://doi.org/10.1039/c0cc04032k

31. Zhao S, Yang W, Lai RY (2011) A folding-based electrochemical aptasensor for detection of vascular endothelial growth factor in human whole blood. Biosens Bioelectron 26(5):2442-2447. https://doi.org/10.1016/j.bios.2010.10.029

32. Iliuk AB, Hu L, Tao WA (2011) Aptamer in bioanalytical applications. Anal Chem 83(12):4440-4452. https://doi.org/10.1021/ ac201057w

33. Zhang H, Zhou L, Zhu Z, Yang C (2016) Recent progress in aptamer-based functional probes for bioanalysis and biomedicine. Chemistry 22(29):9886-9900. https://doi.org/10.1002/chem. 201503543

34. Sun W, Song W, Guo X, Wang Z (2017) Ultrasensitive detection of nucleic acids and proteins using quartz crystal microbalance and surface plasmon resonance sensors based on target-triggering multiple signal amplification strategy. Anal Chim Acta 978:4247. https://doi.org/10.1016/j.aca.2017.04.047

35. Suzuki Y, Yokoyama K (2015) Development of functional fluorescent molecular probes for the detection of biological substances. Biosensors (Basel) 5(2):337-363. https://doi.org/10.3390/bios5 020337

36. Terai T, Nagano T (2013) Small-molecule fluorophores and fluorescent probes for bioimaging. Pflugers Arch 465(3):347-359. https://doi.org/10.1007/s00424-013-1234-Z

37. Shi C, Wu JB, Pan D (2016) Review on near-infrared heptamethine cyanine dyes as theranostic agents for tumor imaging, targeting, and photodynamic therapy. J Biomed Opt 21(5):50901. https://doi.org/10.1117/1.JBO.21.5.050901

Publisher's note Springer Nature remains neutral with regard to jurisdictional claims in published maps and institutional affiliations. 